\title{
Neuromythen sind zu Beginn des Lehramtsstudiums prävalent und unabhängig vom Wissen über das menschliche Gehirn
}

\author{
Georg Krammer (D) - Stephan E. Vogel • Tugba Yardimci • \\ Roland H. Grabner
}

Angenommen: 19. März 2019 / Online publiziert: 8. April 2019

(C) Der/die Autor(en) 2019

Zusammenfassung Der Transfer von neurowissenschaftlichen Befunden in die schulische Praxis wurde begleitet von der Entstehung von Fehlkonzepten über das menschliche Gehirn, sogenannte Neuromythen. Neuromythen sind unter Lehrkräften weltweit verbreitet. Unklar ist, ob Neuromythen bereits zu Beginn des Lehramtsstudiums bestehen und ob diese im österreichischen Bildungssystem eine ähnlich hohe Prävalenz aufweisen wie in anderen Ländern. Die vorliegende Studie untersucht, ob österreichische Lehramtsstudierende ihre Ausbildung bereits mit dem Glauben an Neuromythen beginnen. Darüber hinaus soll die Rolle des Wissens über das menschliche Gehirn für die Prävalenz von Neuromythen kritisch hinterfragt werden. 582 Lehramtsstudierende bearbeiteten zu Beginn ihres Lehramtsstudiums 40 Aussagen über das menschliche Gehirn; 20 dieser Aussagen waren Neuromythen und 20 korrekte Aussagen über das menschliche Gehirn, sogenannte Neurofakten. Es zeigte sich, dass bereits zu Beginn des Lehramtsstudiums mehrere Neuromythen eine hohe Prävalenz aufwiesen. Analog zu anderen Ländern bezogen sich die prävalentesten Neuromythen auf Lerntypen und auf ein getrenntes Arbeiten der Gehirnhälften. Darüber hinaus wurde gefunden, dass Neuromythen kein eindimensionales Konstrukt abbilden. Schließlich zeigte sich, dass das Wissen über das menschliche Gehirn nicht mit dem Glauben an Neuromythen zusammenhing. Da Neuromythen bereits zu Lehramtsstudienbeginn stark vertreten sind, sollte die Lehramtsausbildung diese auch auflösen. Hierfür scheint es ratsam zu sein, nicht nur generell Wissen über das menschliche Gehirn zu vermitteln, sondern Neuromythen gezielt zu thematisieren. Dies sollte insbesondere für jene Neuromythen getan werden, deren Umsetzung zu potentiell schädlichen Schulpraktiken führen könnte.

\footnotetext{
G. Krammer

Institut für Praxislehre und Praxisforschung, Pädagogische Hochschule Steiermark, Graz, Österreich

S. E. Vogel $(\bowtie) \cdot$ T. Yardimci · R. H. Grabner

Educational Neuroscience, Institut für Psychologie, Karl-Franzens-Universität Graz, Graz, Österreich

E-Mail: stephan.vogel@uni-graz.at
} 
Schlüsselwörter Neuromythen · Prävalenz $\cdot$ Lehrerinnenbildung und Lehrerbildung $\cdot$ Neurofakten $\cdot$ Faktorstruktur

\title{
Neuromyths are prevalent and independent of the knowledge of the human brain at the beginning of teacher education
}

\begin{abstract}
Transferring neuroscientific insights into education has created misconceptions of the human brain, i.e. neuromyths. Studies suggest that neuromyths are widely spread among teachers world-wide. However, it is unclear whether neuromyths already exist at the beginning of teacher education and whether they have a similar prevalence in Austria compared to other countries. The aim of the present study is to address these questions. In addition, this study aims to scrutinize the relationship between knowledge about the human brain and believing in neuromyths. 582 Austrian teacher education students responded to 40 statements of the human brain. Of these 40 statements, 20 were neuromyths and 20 were correct statements about the brain, i.e. neurofacts. Results showed that some neuromyths have a high prevalence already at the beginning of teacher education. Similar to previous findings in other countries, the most widely believed neuromyths were related to learning styles and to a disjoint functioning of the brain hemispheres. Furthermore, neuromyths did not form a unidimensional factor, as assumed-but not tested-by prior studies. Finally, results suggest that the knowledge about the human brain was not related to believing in neuromyths. To summarize, neuromyths are already prevalent at the beginning of teacher education. Teacher education should therefore take care to dispel these neuromyths by addressing neuromyths directly instead of only fostering general knowledge about the human brain. This holds particularly true for those neuromyths that could be potentially harmful when implemented in educational practices.
\end{abstract}

Keywords Neuromyths $\cdot$ Prevalence $\cdot$ Teacher education $\cdot$ Neurofacts $\cdot$ Factorial structure

\section{Einleitung}

Mit der zunehmenden Zahl an neurowissenschaftlichen Studien in der empirischen Lehr- und Lernforschung gewinnen Befunde aus der Hirnforschung zunehmend an Bedeutung für Lehrpersonen. Insbesondere sind Lehrkräfte häufig mit einer Vielzahl von Aussagen darüber konfrontiert, wie das menschliche Gehirn lerne und welche Implikationen dies für ,gehirngerechten“ Unterricht habe. Viele dieser Aussagen sind allerdings wissenschaftlich nicht haltbar und werden als Neuromythen bezeichnet. Im Kontext von Lehren und Lernen wurde der Begriff Neuromythos erstmalig von der OECD erwähnt und definiert als „Fehlkonzept entstanden aus einem Missverstehen, Missinterpretieren oder Fehlzitieren von wissenschaftlich fundierten Fakten (aus der Hirnforschung), um die Anwendung von Hirnforschung in Bildung und 
anderen Kontexten zu rechtfertigen“( OECD 2002) $)^{1}$. Untersuchungen zur Prävalenz von Neuromythen zeigen, dass bestimmte Neuromythen unter Lehrkräften weit verbreitet sind (für einen Übersicht: Howard-Jones 2014). Derzeit liegen jedoch noch keine Befunde vor, inwieweit das österreichische Bildungssystem davon betroffen ist und ob Neuromythen bereits zu Beginn des Lehramtsstudiums bestehen.

Die vorliegende Studie adressiert Neuromythen und somit falsche Annahmen über Lehren und Lernen, die vermeintlich mit neurowissenschaftlichen Befunden begründet werden. Kritikerinnen und Kritiker haben hinterfragt, wie relevant neurowissenschaftliche Befunde für die empirische Lehr- und Lernforschung sein können, betonen aber auch in diesem Kontext die Notwendigkeit für Evidenzbasierung (für eine kritische Auseinandersetzung s. Bowers 2016). Neuromythen im Bildungssystem widersprechen - gleich wie Mythen zu Bildung (s. Harmes et al. 2015) dem Grundgedanken eines evidenzorientierten und evidenzbasierten Bildungssystems. Darüber hinaus zeigen neuere Studien auf, dass es wenige aber doch Neuromythen gibt, die zwischen guten und weniger guten Lehrerinnen und Lehrern differenzieren. Es handelt sich hierbei um Neuromythen, die Lernenden Bildbarkeit absprechen (Horvath et al. 2018). Weiters dürfte der Glaube an Neuromythen zu einer ineffektiven Bindung von bildungsbezogenen Ressourcen führen (Howard-Jones und Fenton 2012). Vor diesem Hintergrund rückt die Debatte zur Evidenzbasierung des Bildungssystems noch stärker in den Vordergrund, da durch den Glauben an Neuromythen negative Konsequenzen für Lernende entstehen können.

Auf der Suche nach protektiven Faktoren gegenüber Neuromythen wurde aufgezeigt, dass das wissenschaftlich korrekte Wissen über das Gehirn (sog. Neurofakten) mit der Zustimmung zu Neuromythen positiv zusammenhängt (Dekker et al. 2012; Ferrero et al. 2016). Je höher das wissenschaftlich korrekte Wissen über das Gehirn ausgeprägt war, desto eher stimmten Lehrkräfte diversen Neuromythen zu. Diese Befunde stehen in Widerspruch zu Studien an Lehramtsstudierenden, in denen ein höheres Wissen über Neurofakten mit einer niedrigeren Zustimmung zu Neuromythen einherging (Howard-Jones et al. 2009; Papadatou-Pastou et al. 2017). Vor dem Hintergrund dieser widersprüchlichen Befundlage ist es notwendig, die Rolle des Wissens über Neurofakten für Neuromythen weiter zu untersuchen. Erst wenn die Bedeutung der Neurofakten geklärt ist, kann eine wissenschaftlich fundierte Empfehlung abgegeben werden, ob korrektes Wissen über das menschliche Gehirn ein möglicher protektiver Faktor gegen Neuromythen sein kann.

\subsection{Wie hoch ist die Prävalenz von Neuromythen in der schulischen Praxis?}

Neuromythen sind in der schulischen Praxis weltweit verbreitet (Dekker et al. 2012; Deligiannidi und Howard-Jones 2015; Düvel et al. 2017; Ferrero et al. 2016; Howard-Jones 2014; Howard-Jones et al. 2009; Karakus et al. 2015; Macdonald et al. 2017; Pei et al. 2015; Rato et al. 2013; Simmonds 2014). In einem Übersichtsartikel greift Howard-Jones (2014) sieben populäre Neuromythen im internationalen

\footnotetext{
1 Im Original: „Misconception generated by a misunderstanding, a misreading or a misquoting of facts scientifically established (by brain research) to make a case for use of brain research in education and other contexts" (OECD 2002, S. 111).
} 
Tab. 1 Zustimmungsrate von Lehrkräften zu sieben populären Neuromythen in fünf Ländern entnommen von Howard-Jones (2014), ergänzt um die Zustimmungsrate der Lehramtsstudierenden aus der vorliegenden Studie (Österreich)

\begin{tabular}{|c|c|c|c|c|c|c|c|}
\hline Neuromythen & $\begin{array}{l}\text { Großbri- } \\
\text { tannien } \\
{[\%]}\end{array}$ & $\begin{array}{l}\text { Nieder- } \\
\text { lande } \\
{[\%]}\end{array}$ & $\begin{array}{l}\text { Türkei } \\
{[\%]}\end{array}$ & $\begin{array}{l}\text { Griechen- } \\
\text { land } \\
{[\%]}\end{array}$ & $\begin{array}{l}\text { China } \\
{[\%]}\end{array}$ & $\begin{array}{l}\text { Gesamt } \\
{[\%]}\end{array}$ & $\begin{array}{l}\text { Öster- } \\
\text { reich } \\
{[\%]}\end{array}$ \\
\hline $\begin{array}{l}\text { Wir nutzen nur } 10 \% \text { unseres } \\
\text { Gehirns }\end{array}$ & 48 & 46 & 50 & 43 & 59 & 50 & 43 \\
\hline $\begin{array}{l}\text { Schüler lernen besser, wenn ih- } \\
\text { nen Informationen entsprechend } \\
\text { ihrem Lerntyp (z. B. visuell, au- } \\
\text { ditiv, kinästhetisch) vermittelt } \\
\text { werden }\end{array}$ & 93 & 96 & 97 & 96 & 97 & 96 & 97 \\
\hline $\begin{array}{l}\text { Kurzzeitige Koordinationsübun- } \\
\text { gen können die Integration zwi- } \\
\text { schen linker und rechter Gehirn- } \\
\text { hälfte verbessern }\end{array}$ & 88 & 82 & 72 & 60 & 84 & 76 & 88 \\
\hline $\begin{array}{l}\text { Unterschiede in der Gehirndo- } \\
\text { minanz (linke oder rechte Ge- } \\
\text { hirnhälfte) können individuelle } \\
\text { Unterschiede zwischen Lernen- } \\
\text { den erklären }\end{array}$ & 91 & 86 & 79 & 74 & 71 & 79 & 82 \\
\hline $\begin{array}{l}\text { Kinder sind weniger aufnahme- } \\
\text { fähig nachdem sie zuckerhaltige } \\
\text { Getränke und/oder Snacks zu } \\
\text { sich genommen haben }\end{array}$ & 57 & 55 & 44 & 46 & 62 & 52 & 40 \\
\hline $\begin{array}{l}\text { Sobald Schüler nicht genug Was- } \\
\text { ser (=6-8 Gläser pro Tag) zu sich } \\
\text { nehmen, schrumpft das Gehirn }\end{array}$ & 29 & 16 & 25 & 11 & 5 & 17 & 4 \\
\hline $\begin{array}{l}\text { Lernschwierigkeiten die in Ver- } \\
\text { bindung mit entwicklungsbe- } \\
\text { dingten Unterschieden in der } \\
\text { Gehirnfunktion stehen, können } \\
\text { nicht durch Bildung korrigiert } \\
\text { werden }\end{array}$ & 16 & 19 & 22 & 33 & 50 & 30 & 19 \\
\hline
\end{tabular}

Vergleich heraus. Diese sieben Neuromythen und ihre Zustimmungsraten in fünf Ländern sind in Tab. 1 aufgelistet.

Der Neuromythos mit der höchsten Prävalenz bezieht sich auf „Lerntypen“, nämlich, dass Schülerinnen und Schüler besser lernen, wenn ihnen Informationen entsprechend ihres Lerntyps (z.B. visuell, auditiv, kinästhetisch) vermittelt werden. Dem Neuromythos der Lerntypen liegt vermutlich der Neurofakt zu Grunde, dass visuelle, auditive und kinästhetische Informationen in unterschiedlichen Gehirnregionen verarbeitet werden (Dekker et al. 2012; Howard-Jones 2014). Trotz Mangel an wissenschaftlicher Evidenz und solider theoretischer Fundierung (für eine Zusammenfassung der Kritik s. An und Carr 2017; Pashler et al. 2008), hat sich der Neuromythos der Lerntypen in der schulischen Praxis durchgesetzt. Konkret zeigt Howard-Jones (2014), dass 93\% der Lehrkräfte in Großbritannien, $96 \%$ in den Niederlanden, $97 \%$ in der Türkei, $96 \%$ in Griechenland und $97 \%$ in China dem Neuromythos der Lerntypen zustimmen. Folglich wird der Neuromythos der Lerntypen in der schulischen Praxis auch häufig umgesetzt (Simmonds 2014). 
Im allgemeinen zeigen sich wenige Unterschiede in der Prävalenz von Neuromythen im internationalen Vergleich (z.B. Großbritannien vs. Niederlande: Dekker et al. 2012). Dies deutet darauf hin, dass bestimmte Neuromythen ein generelles Phänomen darstellen und unabhängig von Bildungssystemen und kulturellen Einflüssen sind. Gleichzeitig wurden bei manchen Neuromythen Unterschiede zwischen Ländern gefunden. Diese Unterschiede können vermutlich auf länderspezifische, kommerziell erwerbbare Programme zurückgeführt werden. Als Beispiel sei „,braingym“ genannt, welches vermutlich dazu führte, dass Großbritannien höhere Prävalenzen für Neuromythen, basierend auf einer getrennten neuronalen Verarbeitung der Gehirnhälften, aufweist als andere Länder (Dekker et al. 2012; Simmonds 2014). Zur Prävalenz von Neuromythen im österreichischen Bildungssystem liegen bislang noch keine Ergebnisse vor.

Neuere Studien setzen die Prävalenz von Neuromythen in Bezug zur allgemeinen Bevölkerung, oder weisen auf die Prävalenz in der Ausbildung hin. Zum Beispiel fanden Macdonald et al. (2017) für die USA eine mittlere Zustimmungsrate von $68 \%$ in der allgemeinen Bevölkerung gegenüber $56 \%$ unter Lehrkräften. Weiters wurde berichtet, dass bereits unter Lehramtsstudierenden Neuromythen eine hohe Prävalenz aufweisen (Düvel et al. 2017; Howard-Jones et al. 2009; Papadatou-Pastou et al. 2017), und dass selbst darauf ausgerichtete Lehrveranstaltungen diese Prävalenz nur gering beeinflussen können (Im et al. 2018). Bezogen auf die Ausbildung von Lehrkräften ist derzeit unklar, mit welchen Fehlkonzepten und welchen Vorwissen über das lernende Gehirn die Ausbildung begonnen wird.

\subsection{Wie hängen Neuromythen mit Neurofakten zusammen?}

Neben der Prävalenz von Neuromythen haben Studien wiederholt untersucht, mit welchen Faktoren Neuromythen einhergehen. Als stärkster Faktor hat sich das Wissen über Neurofakten herausgestellt. Einzelne Studien an Lehramtsstudierenden haben erwartungsgemäß gezeigt, dass ein höheres Wissen über Neurofakten mit einer niedrigeren Zustimmung zu Neuromythen einhergeht $(r=-0,43$ bzw. unter Kontrolle von soziodemographischen Variablen: $\beta=-0,34$; Howard-Jones et al. 2009 bzw. Papadatou-Pastou et al. 2017). Dem gegenüber stehen allerdings Befunde an Lehrkräften, dass unter Kontrolle von soziodemographischen Variablen ein höheres Wissen über Neurofakten mit einer höheren Zustimmung zu Neuromythen einhergeht ( $\beta=0,31$ bzw. $\beta=0,24$; Dekker et al. 2012 bzw. Ferrero et al. 2016). Dieses unerwartete Ergebnis wird als überraschend kommentiert (z. B. Macdonald et al. 2017) und als Zeichen dafür interpretiert, dass Lehrkräfte zwischen falschen (Neuromythen) und korrekten (Neurofakten) Aussagen über das Gehirn kaum differenzieren können.

Interpretationen der Zusammenhänge zwischen Neuromythen und dem Wissen über Neurofakten können aus mehreren Gründen problematisch sein. Einerseits sind die Aussagen der Studien rein korrelativ und lassen daher die Kausalität des Zusammenhangs offen. Andererseits können sie dazu führen, dass man sich in der praktischen Umsetzung dagegen ausspricht mehr Wissen über Neurofakten zu vermitteln. Die Widersprüchlichkeit der Befunde könnte allerdings auch ein methodisches Artefakt darstellen, da die bisherigen Studien alle vorgegebenen Neuromythen 
bzw. Neurofakten a priori als jeweils ein Konstrukt behandelten, ohne dies jedoch empirisch zu überprüfen.

\subsection{Faktorstruktur von Neuromythen und Neurofakten}

Ein grundlegender methodischer Aspekt, der nur in wenigen Studien berücksichtigt wird, ist die Zulässigkeit der Bildung eines Gesamtwerts für Neuromythen bzw. Neurofakten. Werden Neuromythen bzw. Neurofakten nicht mehr einzeln betrachtet, sondern für weitere Berechnungen wie Wissenstests zu Gesamtwerten aggregiert, so impliziert dies theoretische und praktische Annahmen. Aus theoretischer Sicht wird davon ausgegangen, dass die Neuromythen bzw. Neurofakten jeweils ein eindimensionales Konstrukt abbilden. Praktisch wird impliziert, dass den Neuromythen bzw. Neurofakten jeweils eine eindimensionale Faktorstruktur zugrunde liegt, die die Bildung eines Gesamtwerts erlaubt (Wright und Stone 1979).

Bisher ist uns nur die Studie von Macdonald et al. (2017) bekannt, in der die Faktorstruktur von Neuromythen bzw. Neurofakten analysiert wurde. Macdonald et al. (2017) gaben Personen aus der Allgemeinbevölkerung, Lehrkräften und neurowissenschaftlichen Expertinnen und Experten 15 Neuromythen und 17 Neurofakten vor. Nur 9 Neuromythen ließen sich zu einem Faktor zusammenfassen. Innerhalb der übrigen 6 Neuromythen und 17 Neurofakten konnte keine interpretierbare Faktorstruktur gefunden werden. Für die weiteren Analysen wurde daher nur ein Gesamtwert aus den 9 Neuromythen gebildet. Diese Befunde zur Faktorstruktur werfen ein kritisches Licht auf Studien zum Zusammenhang von Neuromythen und Neurofakten, die ungeprüft von der Eindimensionalität aller vorgegebenen Neuromythen und Neurofakten ausgehen.

Macdonald et al. (2017) verwendeten eine explorative Herangehensweise zur Überprüfung der Faktorstruktur von Neuromythen und Neurofakten. Die Vorgabe von Neuromythen und Neurofakten geht allerdings von einer klaren theoretischen Faktorstruktur aus, nämlich, dass es jeweils einen Faktor für Neuromythen bzw. Neurofakten gibt. Diese Annahme sollte konfirmatorisch überprüft werden.

\subsection{Die vorliegende Studie}

Die vorliegende Studie verfolgt somit drei Ziele. Erstens soll die Prävalenz von Neuromythen zu Beginn des Lehramtsstudiums in Österreich erhoben und mit bisherigen Studien deskriptiv verglichen werden.

Zweitens soll die Rolle des Wissens zu Neurofakten für die Prävalenz von Neuromythen kritisch hinterfragt werden. Die in vorherigen Studien implizierte eindimensionale Faktorstruktur von Neuromythen (Hypothese 1.1) und Neurofakten (Hypothese 1.2) wird daher konfirmatorisch überprüft.

Drittens wird die Annahme, dass ein höheres Wissen einen protektiver Faktor gegen Neuromythen bei Lehramtsstudierenden darstellt (Howard-Jones et al. 2009; Papadatou-Pastou et al. 2017), untersucht. Unter Berücksichtigung der tatsächlich gegebenen Faktorstruktur (s. Hypothesen 1.1 und 1.2) erwarten wir, dass ein höheres Wissen über Neurofakten mit einer niedrigeren Zustimmung zu Neuromythen einhergeht (Hypothese 2). 


\section{Methode}

\subsection{Stichprobe}

Die Datenerhebung lief über zwei Studienjahre. In jedem der beiden Studienjahre wurden Lehramtsstudierende direkt zu Beginn ihres Studiums befragt. Der erste Teil der Erhebung fand im Wintersemester 2016 statt, der zweite Teil im Wintersemester 2017. Um so viele Lehramtsstudierende wie möglich zu Beginn ihres Studiums zu erreichen, wurde in der ersten Einführungsvorlesung des Lehramtsstudiums der Sekundarstufe (laut Curriculum) rekrutiert. Die Studierenden füllten den Fragebogen direkt in der Einführungsvorlesung aus. Die Teilnahme an der Studie war anonym und freiwillig. Die Studie wurde von der lokalen Ethikkommission genehmigt.

Insgesamt nahmen 673 Studierende an der Studie teil, die in Graz (Österreich) an einem Lehramtsstudium der Karl-Franzens-Universität Graz und der Pädagogischen Hochschule Steiermark studierten. Im ersten Schritt wurden jene Studierende ausgeschlossen, die sich nicht im ersten Studiensemester befanden oder keine Angabe zu ihrem Studienfortschritt machten $(n=58)$. Im nächsten Schritt wurden jene Studierende ausgeschlossen, die das Lehramtsstudium nicht als Hauptstudium studierten $(n=32)$. Schließlich wurde eine Person ausgeschlossen, die den Großteil des Fragebogens leer abgegeben hatte. Die finale Stichprobe umfasste somit 582 Lehramtsstudierende des Sekundarstufen-Lehramts im ersten Semester. Die 582 Lehramtsstudierenden waren im Durchschnitt 19,95 $(S D=2,84)$ Jahre alt. Unter ihnen waren $368(\sim 63,2 \%)$ Frauen und $214(\sim 36,8 \%)$ Männer.

Von den 582 Lehramtsstudierenden nahmen $255(\sim 43,7 \%)$ an der ersten Erhebung teil und $327(\sim 56,1 \%)$ an der zweiten. Die Vergleichbarkeit der beiden Stichproben wurde über die Verteilung der relativen Antworthäufigkeiten, des Geschlechts und des Alters geprüft. Die relativen Antworthäufigkeiten der 20 Neuromythen und der 20 Neurofakten wichen im Mittel um 0,07\% ab $(S D=3,18 \%$, Spannweite: $-8,08$ bis $10,69 \%$ ). Alle relativen Antworthäufigkeiten sind in Tab. 3 (siehe Anhang) gesondert für beide Teilstichproben aufgeschlüsselt. Weder das Geschlecht $\left(\chi^{2}[1]=0,23\right.$, $p=0,67$, Cramer $V=0,02$ ) noch das Alter (1. Erhebung: $M=19,83, S D=2,58 ; 2$. Erhebung: $M=20,03, S D=3,03 ; t[579]=-0,84, p=0,40, d=0,07)$ waren mit dem Erhebungszeitpunkt konfundiert. Die beiden Stichproben können somit als vergleichbar betrachtet werden.

\subsection{Material und Prozedere}

Den Lehramtsstudierenden wurden in einem Fragebogen 20 Neuromythen und 20 Neurofakten in einer zufälligen Reihenfolge vorgegeben. Sie konnten zwischen drei Antwortmöglichkeiten wählen: richtig, falsch oder weiß nicht. Der überwiegende Teil dieser Fragen wurde direkt aus der Forschungsarbeit von Dekker et al. (2012) übernommen, ins Deutsche übersetzt und an die spezifischen Sprachkonventionen angepasst. Zusätzlich wurde diese Liste um weitere Mythen und Fakten erweitert. Diese wurden vorwiegend aus einer gemeinsamen Stellungnahme der Nationalen Akademie der Wissenschaften Leopoldina, der Union der deutschen Akademien der Wissenschaften und der Deutschen Akademien der Technikwissenschaften acatech 
zur „Frühkindlichen Sozialisation“ (2014) entnommen. Eine detaillierte Auflistung der Quellen findet sich in Tab. 3 (siehe Anhang).

Vor den Neuromythen und Neurofakten wurden die in der Stichprobenbeschreibung genannten soziodemographischen Daten erhoben.

\subsection{Statistische Auswertungen}

Alle Analysen wurden in $R$ (R Core Team 2018) durchgeführt. Für die Strukturgleichungsmodelle wurde lavaan (Rosseel 2012) verwendet. Die Daten und die R-Skripten für die Analysen sind unter https://osf.io/5tsfv/ verfügbar (Krammer et al. 2019).

\subsubsection{Prävalenz von Neuromythen}

Im ersten Schritt wurden die Prävalenzen der Neuromythen betrachtet. Die Prävalenz entspricht der prozentualen Rate der Zustimmung zu den einzelnen Neuromythen. Diese Prävalenzen wurden deskriptiv mit bisherigen internationalen Studien verglichen.

\subsubsection{Faktorstruktur von Neuromythen und Neurofakten}

Im zweiten Schritt wurde für die Neuromythen und Neurofakten die Faktorstruktur geprüft. Bei Neuromythen wurde die Antwort falsch als richtig kodiert, bei Neurofakten die Antwort richtig. Zuerst wurde mittels Strukturgleichungsmodellen überprüft, ob allen Neuromythen bzw. Neurofakten jeweils ein Faktor zugrunde lag (Hypothese 1.1 und Hypothese 1.2). Sollten sich alle Neuromythen bzw. Neurofakten zu einem Faktor zusammenfassen lassen, so ist eine Aggregation zu jeweils einem Gesamtwert zulässig. Sollte dies nicht der Fall sein, wurde in der Teilstichprobe der zweiten Erhebung - der größeren Teilstichprobe - eine eindimensionale Lösung durch systematisches Ausschließen von Neuromythen bzw. Neurofakten angestrebt. Hierfür wurden iterativ Neuromythen bzw. Neurofakten auf Grund von zu geringer Streuung, zu niedrigen Faktorladungen und schließlich korrelierten Messfehlern ausgeschlossen. Wurde eine eindimensionale Lösung für die Teilstichprobe der zweiten Erhebung gefunden, so wurde diese in der Teilstichprobe der ersten Erhebung kreuzvalidiert. Hielt die eindimensionale Lösung der Kreuzvalidierung stand, wurden für die weiteren Berechnungen die verbliebenen Neuromythen bzw. Neurofakten verwendet, um Faktorwerte zu bilden (EBM). Sollte keine eindimensionale Lösung gefunden werden, wurden keine Gesamtwerte gebildet und die Neuromythen bzw. Neurofakten einzeln betrachtet.

Für die Strukturgleichungsmodelle gingen die Neuromythen bzw. Neurofakten als manifeste Indikatoren in die Analysen ein. Da sie dichotom waren, wurden für die Strukturgleichungsmodelle der WLSMV (weighted least square mean and variance adjusted) Schätzer verwendet (Lei 2009; Savalei und Rhemtulla 2013). Der Modellfit wurde als zufriedenstellend angenommen, wenn die $\chi^{2}$-Statistik nicht signifikant ( $p \geq 0,05$ ) war (Greiff und Heene 2017) und wenn CFI (comparative fit index) $\geq 0,95$ und RMSEA (root mean square error of approximation) $<0,06$ ( Hu und 
Bentler 1999; Marsh et al. 2004). Da die Neuromythen bzw. Neurofakten dichotom in die Strukturgleichungsmodelle eingingen, wurde statt dem sonst üblichen SRMR (standardized root mean square residual) der besser geeignete WRMR (weighted root mean square residual) verwendet, welcher Modellpassung bei Werten kleiner 1 anzeigt (Yu 2002). Modellpassung wurde erst angenommen, wenn alle genannten Kriterien erfüllt waren. Die Signifikanz der Faktorladungen wurde über deren Standardfehler mit einem Signifikanzniveau von 5\% berechnet. Die standardisierten Faktorladungen aller Modelle sind im Tab. 4 im Anhang angeführt.

\subsubsection{Wie hängen Neuromythen mit Neurofakten zusammen?}

Im letzten Schritt wurden die Zusammenhänge zwischen den Neuromythen und Neurofakten analysiert (Hypothese 2). Diese wurden in Abhängigkeit der gewonnenen Erkenntnisse zur Struktur der Neuromythen bzw. Neurofakten berechnet.

\section{Ergebnisse}

\subsection{Prävalenz von Neuromythen}

Eine detaillierte Übersicht der Prävalenzen aller Neuromythen und Neurofakten findet sich in Tab. 2. Die Zustimmungsrate zu Neuromythen lag zwischen 1 und $97 \%$. Für Neurofakten lag die Zustimmungsrate zwischen 18 und $92 \%$. Die prävalentesten Neuromythen waren, dass 1) Schülerinnen und Schüler besser lernen, wenn ihnen Informationen entsprechend ihres jeweiligen Lerntyps vermittelt werden (Lerntypen-Mythos; Zustimmungsrate $=97 \%$ ), 2) kurzzeitige Koordinationsübungen die Integration zwischen den Gehirnhälften verbessern können (KoordinationsübungsMythos; Zustimmungsrate $=88 \%$ ) und 3) Unterricht so gestaltet werden sollte, dass beide Gehirnhälften beansprucht werden (Zustimmungsrate $=86 \%$ ). Andere Neuromythen hingegen wurden fast einstimmig als falsch erkannt, wie z.B. dass das Gehirn im Schlaf nicht aktiv ist (Ablehnungsrate $=95 \%$ ). Teilweise gaben auch größere Teile von Studierenden an, nicht zu wissen ob Neuromythen richtig oder falsch waren. Zum Beispiel beurteilten $46 \%$ die Aussage, dass Nahrungsergänzungsmittel mit Fettsäure (Omega-3, Omega-6) eine positive Auswirkung auf den akademischen Erfolg haben, mit ,weiß nicht“.

Die Prävalenzen der Neuromythen in der vorliegenden Studie sind konsistent mit jenen anderer internationalen Erhebungen. So zeigt sich etwa in Großbritannien eine Zustimmungsrate von $93 \%$ für den Lerntypen-Mythos und eine Zustimmungsrate von $88 \%$ für den Koordinationsübungs-Mythos. Die Prävalenzen der sieben populärsten Neuromythen sind in Tab. 1 gegenübergestellt.

Bezüglich der Neurofakten zeigte sich, dass manche bereits zu Beginn des Lehramtsstudiums bekannt sind und andere nicht. Der Großteil der Lehramtsstudierenden wusste bereits zu Beginn des Studiums, dass es in der Kindheit sensible Phasen gibt, in denen es einfacher ist Dinge zu lernen (Zustimmungsrate $=92 \%$ ). Dahingegen wurden andere Neurofakten von der Mehrheit abgelehnt, wie z. B. dass Jungen größere Gehirne haben als Mädchen (Ablehnungsrate $=63 \%$ ). 
Tab. 2 Relative Häufigkeiten der gewählten Antwortkategorien (richtig, falsch, oder weiß nicht) der Neuromythen und Neurofakten, sortiert jeweils absteigend nach der relativen Häufigkeit der Zustimmung

\begin{tabular}{llllr}
\hline & & $\mathrm{R}$ & $\mathrm{F}$ & $\mathrm{WN}$ \\
\hline 1. & $\begin{array}{l}\text { Schüler lernen besser, wenn ihnen Informationen entsprechend ih- } \\
\text { rem Lerntyp (z. B. visuell, auditiv, kinästhetisch) vermittelt werden }\end{array}$ & 0,97 & 0,01 & 0,02 \\
2. & $\begin{array}{l}\text { Kurzzeitige Koordinationsübungen können die Integration zwi- } \\
\text { schen linker und rechter Gehirnhälfte verbessern }\end{array}$ & 0,88 & 0,05 & 0,08 \\
3. & $\begin{array}{l}\text { Der Unterricht sollte so gestaltet werden, dass beide Gehirnhälften } \\
\text { beansprucht werden }\end{array}$ & 0,86 & 0,02 & 0,12 \\
4. & Unterschiede in der Gehirndominanz (linke oder rechte Gehirn- & 0,82 & 0,03 & 0,15
\end{tabular}
hälfte) können individuelle Unterschiede zwischen Lernenden erklären

5. Übungen der Körper-Augen-Koordination können sich positiv auf die Lesefähigkeit auswirken

6. Es gibt nicht nur eine, sondern mehrere, voneinander unabhängige Intelligenzen, die in unterschiedlichen Gehirnregionen lokalisiert sind

7. Reizreiche Umgebungen verbessern die Gehirnentwicklung von Kindergartenkindern

8. In der Kindheit gibt es kritische Phasen, nach denen bestimmte Dinge nicht mehr gelernt werden können

9. Wir nutzen nur $10 \%$ unseres Gehirns

$0,80 \quad 0,04 \quad 0,16$

$0,60 \quad 0,07 \quad 0,32$

10. Der mehrjährige Schulbesuch macht die Kinder weniger kreativ. Am kreativsten sind Kinder vor der Einschulung

11. Kinder sind weniger aufnahmefähig nachdem sie zuckerhaltige Getränke und/oder Snacks zu sich genommen haben

12. Es ist wissenschaftlich erwiesen, dass Fettsäure (Omega-3, Omega-6) enthaltende Nahrungsergänzungsmittel eine positive Auswirkung auf den akademischen Erfolg haben

13. Kinder müssen ihre Muttersprache (L1) erwerben, bevor sie weitere Sprachen (L2, L3) lernen. Ist dies nicht der Fall, wird keine der Sprachen vollständig erworben

14. Die Gehirne von Jungen und Mädchen entwickeln sich gleich schnell

15. Lernschwierigkeiten, die in Verbindung mit entwicklungsbedingten Unterschieden in der Gehirnfunktion stehen, können nicht durch Bildung korrigiert werden

16. Intelligenz wird vererbt und kann nicht durch Erfahrung oder Umwelteinflüsse verändert werden

17. Hochbegabte brauchen nicht zu lernen, um gute schulische Leistungen $\mathrm{zu}$ erbringen

18. Sobald Schüler nicht genug Wasser (=6-8 Gläser pro Tag) zu sich nehmen, schrumpft das Gehirn

$0,57 \quad 0,16 \quad 0,27$

$0,51 \quad 0,29 \quad 0,20$

$0,43 \quad 0,38 \quad 0,19$

$0,42 \quad 0,32 \quad 0,26$

$0,40 \quad 0,34 \quad 0,26$

$0,38 \quad 0,16 \quad 0,46$

$0,35 \quad 0,53 \quad 0,12$

$0,19 \quad 0,54 \quad 0,26$

$0,19 \quad 0,49 \quad 0,32$

$0,06 \quad 0,85 \quad 0,08$

$0,05 \quad 0,87 \quad 0,08$

$0,04 \quad 0,75 \quad 0,21$

19. Die Gehirnentwicklung ist zwischen dem 11.-12. Lebensjahr $\quad 0,04 \quad 0,66 \quad 0,29$ abgeschlossen

\begin{tabular}{llrrr}
$20 .^{\mathrm{b}}$ & Wenn wir schlafen ist das Gehirn nicht aktiv & 0,01 & 0,95 & 0,04 \\
\hline $1 . \quad \begin{array}{l}\text { In der Kindheit gibt es sensible Phasen in denen es einfacher ist } \\
\text { Dinge zu lernen }\end{array}$ & 0,92 & 0,02 & 0,06 \\
$2 .^{\mathrm{a}} \quad \begin{array}{l}\text { Lernfähigkeit besteht in einigen Funktionsbereichen ein Leben } \\
\text { lang }\end{array}$ & 0,91 & 0,03 & 0,06 \\
\hline
\end{tabular}


Tab. 2 (Fortsetzung)

\begin{tabular}{|c|c|c|c|c|}
\hline & & $\mathrm{R}$ & $\mathrm{F}$ & WN \\
\hline 3. ${ }^{a}$ & $\begin{array}{l}\text { Information ist in einem Netzwerk von Nervenzellen, das über das } \\
\text { Gehirn verteilt ist gespeichert }\end{array}$ & 0,86 & 0,04 & 0,10 \\
\hline $4 .^{\mathrm{a}}$ & $\begin{array}{l}\text { Die Entstehung neuer Gehirnverbindungen ist bis ins hohe Alter } \\
\text { möglich }\end{array}$ & 0,85 & 0,04 & 0,10 \\
\hline $5 .^{\text {a }}$ & $\begin{array}{l}\text { Wiederholtes und langfristiges Training bestimmter geistiger Ab- } \\
\text { läufe kann die Form und Struktur mancher Gehirnregionen verän- } \\
\text { dern }\end{array}$ & 0,84 & 0,06 & 0,11 \\
\hline $6 .^{\mathrm{a}}$ & Wir nutzen unser Gehirn $24 \mathrm{~h}$ am Tag & 0,83 & 0,14 & 0,03 \\
\hline 7. ${ }^{\mathrm{a}}$ & $\begin{array}{l}\text { Lern- und Gedächtnisleistungen können mit steigendem Inhalts- } \\
\text { wissen erhöht werden }\end{array}$ & 0,80 & 0,06 & 0,13 \\
\hline $8 .^{\mathrm{a}}$ & $\begin{array}{l}\text { Die normale Entwicklung des Gehirns bedingt sowohl die Bildung } \\
\text { als auch das Absterben von Gehirnzellen }\end{array}$ & 0,71 & 0,06 & 0,23 \\
\hline 9. ${ }^{\text {a }}$ & $\begin{array}{l}\text { Lernen geschieht im Gehirn durch die Veränderung neuronaler } \\
\text { Verbindungen }\end{array}$ & 0,70 & 0,05 & 0,24 \\
\hline 10. & $\begin{array}{l}\text { Anstrengende Bewegung kann die geistige Leistungsfähigkeit } \\
\text { fördern }\end{array}$ & 0,68 & 0,11 & 0,21 \\
\hline $11 .^{\mathrm{a}}$ & $\begin{array}{l}\text { Durch Verhaltensinterventionen lassen sich ungewöhnliche Ent- } \\
\text { wicklungsverläufe verändern }\end{array}$ & 0,61 & 0,06 & 0,32 \\
\hline 12. & $\begin{array}{l}\text { Der zirkadiane Rhythmus „Schlaf-Wach-Rhythmus“ verändert sich } \\
\text { während der Pubertät, was dazu führt, dass SchülerInnen während } \\
\text { der ersten Stunden des Schultages müde sind }\end{array}$ & 0,59 & 0,14 & 0,27 \\
\hline $13 .^{\mathrm{a}}$ & $\begin{array}{l}\text { Die Funktionen von beschädigten Gehirnregionen können von } \\
\text { anderen Gehirnregionen übernommen werden }\end{array}$ & 0,53 & 0,27 & 0,20 \\
\hline $14 .^{\mathrm{a}}$ & $\begin{array}{l}\text { Existierende Synapsen werden durch Erfahrungen entweder ver- } \\
\text { stärkt oder eliminiert }\end{array}$ & 0,52 & 0,13 & 0,34 \\
\hline 15. & $\begin{array}{l}\text { Das Auslassen des Frühstücks kann den akademischen Erfolg } \\
\text { beeinflussen }\end{array}$ & 0,43 & 0,35 & 0,23 \\
\hline 16. & Lernen erfolgt nicht durch die Bildung neuer Nervenzellen & 0,38 & 0,35 & 0,26 \\
\hline 17. & $\begin{array}{l}\text { Gehirnverletzungen im frühen Kindesalter haben vergleichsweise } \\
\text { negativere Folgen, als solche im Vorschulalter }\end{array}$ & 0,38 & 0,19 & 0,42 \\
\hline 18. & $\begin{array}{l}\text { Regelmäßiger Koffeinkonsum verringert die Wachsamkeit/ } \\
\text { Aufmerksamkeit }\end{array}$ & 0,36 & 0,29 & 0,34 \\
\hline 19. & Die linke und rechte Gehirnhälfte arbeiten immer zusammen & 0,30 & 0,56 & 0,14 \\
\hline 20. & Das Gehirn von Jungs ist größer als das Gehirn von Mädchen & 0,18 & 0,63 & 0,18 \\
\hline
\end{tabular}

Die relative Häufigkeit der korrekten Antwortkategorie ist kursiv gesetzt $R$ richtig, $F$ falsch, $W N$ weiß nicht

${ }^{a}$ Die mit ${ }^{a}$ markierten Neurofakten ließen sich zu einem Faktor zusammenfassen

${ }^{b}$ Der mit ${ }^{b}$ markierte Neuromythos war jener, dessen Ablehnung mit einem höheren Wissen zu Neurofakten einherging als dessen Annahme

\subsection{Faktorstruktur von Neuromythen und Neurofakten}

Im zweiten Schritt wurde für die Neuromythen und Neurofakten konfirmatorisch geprüft, ob sich diese zu jeweils einem Faktor zusammenfassen lassen. Ein Strukturgleichungsmodell mit einer eindimensionalen Lösung für die 20 Neuromythen konnte nicht geschätzt werden. Innerhalb der 20 Neuromythen wiesen die drei prävalentesten Neuromythen sehr geringe Streuungen auf (Ablehnungsrate 1-5\%; 
Neuromythen \#1, \#2, \#3). Nach Ausschluss dieser drei Neuromythen konnte das Modell über die gesamte Stichprobe geschätzt werden. Dieses Modell passte allerdings nicht auf die Daten $\left(\chi^{2}[119]=134,421, p=0,158, \mathrm{CFI}=0,889\right.$, RMSEA =0,015, $\mathrm{WRMR}=0,909$; s. Hypothese 1.1). Die 20 Neurofakten ergaben ebenso keine eindimensionale Lösung in der gesamten Stichprobe $\left(\chi^{2}[170]=232,166, p=0,001\right.$, $\mathrm{CFI}=0,833$, RMSEA =0,025, WRMR =1,024; s. Hypothese 1.2). Da sich weder die Neuromythen noch die Neurofakten zu jeweils einem Faktor in der Gesamtstichprobe zusammenfassen ließen, wurde eine mögliche eindimensionale Lösung an der Teilstichprobe der zweiten Erhebung extrahiert und an der Teilstichprobe der ersten Erhebung kreuzvalidiert.

\subsubsection{Neuromythen}

Nach Ausschluss der drei prävalentesten Neuromythen (siehe oben) konnte das Strukturgleichungsmodell mit einer eindimensionalen Lösung für die Teilstichprobe der zweiten Erhebung geschätzt werden. Das Modell passte jedoch nicht auf die Daten $\left(\chi^{2}[119]=128,471, p=0,261, \mathrm{CFI}=0,868, \mathrm{RMSEA}=0,016, \mathrm{WRMR}=0,893\right)$. Durch den weiteren Ausschluss von drei Neuromythen mit den niedrigsten Faktorladungen (Neuromythen \#7, \#14, \#20) konnte ein zufriedenstellender Modellfit erreicht werden $\left(\chi^{2}[77]=77,787, p=0,453, \mathrm{CFI}=0,989\right.$, RMSEA $=0,006$, WRMR $=0,818$ ). Die Kreuzvalidierung in der Teilstichprobe der ersten Erhebung konnte diese eindimensionale Lösung allerdings nicht bestätigen $\left(\chi^{2}[77]=87,753\right.$, $p=0,189, \mathrm{CFI}=0,790, \mathrm{RMSEA}=0,023$, WRMR =0,880). Die Neuromythen waren somit nicht zu einem gemeinsamen Faktor zusammenfassbar.

\subsubsection{Neurofakten}

Die 20 Neurofakten ergaben ebenso keine eindimensionale Lösung in der Teilstichprobe der zweiten Erhebung $\left(\chi^{2}[170]=199,795, p=0,059\right.$, CFI $=0,768$, RMSEA $=0,023$, WRMR $=0,954)$. Vor allem der CFI wies auf einen unzureichenden Modellfit hin. Deshalb wurden zuerst Neurofakten mit nicht signifikanten Faktorladungen ausgeschlossen (Neurofakten \#1, \#10, \#12, \#15, \#16, \#17, \#18, \#19, \#20). Dadurch konnte ein zufriedenstellender Modellfit durch den Ausschluss von neun Neurofakten erzielt werden $\left(\chi^{2}[44]=45,178, p=0,423, \mathrm{CFI}=0,987\right.$, RMSEA $=0,009$, WRMR =0,764). Die Kreuzvalidierung an der Teilstichprobe der ersten Erhebung bestätigte diese eindimensionale Lösung ( $\chi^{2}[44]=49,552$, $p=0,261, \mathrm{CFI}=0,970, \mathrm{RMSEA}=0,022, \mathrm{WRMR}=0,791)$.

Diese gefundene Faktorstruktur umfasste elf Neurofakten (markiert mit a in Tab. 2). In der gesamten Stichprobe zeigte sich ein zufriedenstellender Modellfit dieser eindimensionalen Lösung $\left(\chi^{2}[44]=54,654, p=0,130\right.$, CFI $=0,960$, RMSEA $=0,020$, WRMR $=0,839)$. Alle Faktorladungen $(0,262<\lambda<0,641)$ waren signifikant und die interne Konsistenz des Faktors war zufriedenstellend (Cronbachs $\alpha=0,69)^{2}$. Es ist daher zulässig aus diesen elf Neurofakten einen Gesamtwert zu bilden.

\footnotetext{
${ }^{2}$ Basierend auf polychorischen Korrelationen.
} 


\subsection{Wie hängen Neuromythen mit Neurofakten zusammen?}

Im letzten Schritt wurde analysiert, wie das Wissen über Neurofakten mit der $\mathrm{Zu}$ stimmung zu Neuromythen zusammenhing (Hypothese 2). Da für die Neuromythen keine eindimensionale Faktorstruktur gefunden werden konnte, wurden diese einzeln betrachtet. Für die Neurofakten wurden die Faktorwerte aus der gefundenen und kreuzvalidierten eindimensionalen Lösung verwendet und die mittleren Unterschiede im Wissen zu Neurofakten zwischen Lehramtsstudierenden, die die Neuromythen ablehnten und annahmen, verglichen (Bonferroni korrigierte t-tests: $\alpha=0,05 / 20=0,0025$ ). Aufgrund von teilweise sehr ungleichen Zellenbesetzungen wurden gefundene Unterschiede im non-parametrischen Vergleich auf Robustheit geprüft (Mann-Whitney-U Test).

Für 19 der 20 Neuromythen konnte kein Unterschied im Wissen zu Neurofakten gefunden werden zwischen jenen Lehramtsstudierenden, die den Neuromythen zustimmten, und jenen, die die Mythen ablehnten $(-1,400 \leq t \mathrm{~s} \leq 1,609$, $0,115 \leq p s \leq 0,925,-0,41 \leq d s \leq 0,58)$. Der Neuromythos mit dem größten mittleren Unterschied war signifikant $(\mathrm{t}[31,987]=-3,83, p<0,001, d=0,72 ; W=10.656$, $p<0,001)$. Für diesen Neuromythos verfügten jene Lehramtsstudieren, die ihn ablehnten, über ein höheres Wissen zu Neurofakten, als jene die ihm zustimmten. Der betreffende Neuromythos ist in Tab. 2 mit $^{\mathrm{b}}$ markiert.

Zur Vergleichbarkeit der Ergebnisse mit bisherigen Studien, wurden zusätzliche Analysen ohne die Berücksichtigung der Faktorstruktur durchgeführt. Das Ergebnis dieser Analyse ist direkt vergleichbar mit jenen Studien, die a priori eine eindimensionale Faktorstruktur annahmen (Dekker et al. 2012; Ferrero et al. 2016; HowardJones et al. 2009; Papadatou-Pastou et al. 2017). Es zeigte sich, dass je mehr Neurofakten gewusst wurden, desto mehr Neuromythen richtigerweise als falsch erkannt wurden $(r=0,22, p<0,001)$. Es zeigte sich aber auch, dass je mehr Neurofakten gewusst wurden, desto mehr Neuromythen auch fälschlicherweise geglaubt wurden $(r=0,31, p<0,001)$. Alle manifesten Zusammenhänge ohne Berücksichtigung einer Faktorstruktur sind in Tab. 5 im Anhang angeführt.

\section{Diskussion}

\subsection{Prävalenz von Neuromythen}

Die Ergebnisse der Studie zeigen, dass Neuromythen auch im österreichischen Bildungssystem weit verbreitet sind. Die prävalentesten Neuromythen unter Lehramtsstudierenden zu Beginn ihrer Ausbildung beziehen sich auf 1) Lerntypen, 2) Koordinationsübungen zur Integration zwischen den Gehirnhälften, 3) Berücksichtigung beider Gehirnhälften bei Unterrichtsgestaltung und 4) Gehirndominanz als individueller Unterschied zwischen Lernenden. Mit der Ausnahme des 3. Mythos sind dies auch jene Neuromythen, die im internationalen Vergleich am weitesten verbreitet sind (s. Tab. 1 bzw. Howard-Jones 2014).

Andere Neuromythen sind vergleichsweise weniger weit verbreitet unter österreichischen Lehramtsstudierenden, wie zum Beispiel, dass das Gehirn durch zu 
geringen Wasserkonsum schrumpfen kann (Zustimmungsrate 4\%). Diese korrekte Ablehnung findet sich auch im internationalen Vergleich, obwohl die Zustimmungsrate in einigen Ländern durchaus höher ausfällt als bei österreichischen Lehramtsstudierenden (z.B. Türkei: 25\%). Diese Befunde deuten einerseits darauf hin, dass der Glaube an Neuromythen ein generelles Phänomen ist und vermutlich unabhängig von Bildungssystemen und diversen kulturellen Einflüssen entsteht, andererseits gibt es dennoch geringfüge Unterschiede in der Ausprägung diverser Mythen in verschiedenen Ländern.

Gleich wie in anderen Ländern, scheint unter österreichischen Lehramtsstudierenden der Glauben an eine getrennte neuronale Verarbeitung in den Gehirnhälften den prävalentesten Neuromythen zugrunde zu liegen. Diese grundlegende Fehlkonzeption wird dadurch bestärkt, dass der Neurofakt, dass beide Gehirnhälften immer zusammenarbeiten, nur von knapp einem Drittel der Lehramtsstudierenden als korrekt angenommen wurde.

Die Vermittlung von neurowissenschaftlichen Befunden für die schulische Praxis sollte daher nicht nur darauf beschränkt sein, wie das menschliche Gehirn lernt, sondern auch den Aufbau und die grundlegende Funktionsfähigkeit des menschlichen Gehirns thematisieren. Bisher wurde die Implementierung von neurowissenschaftlichen Trainings überwiegend für Lehrkräfte beschrieben (z. B. Dubinsky et al. 2013; Hardiman et al. 2012; Thomas 2013). Analog könnten neurowissenschaftliche Trainings bereits im Lehramt angeboten werden. Trainings für Lehrkräfte und Lehramtsstudierende sollten von neurowissenschaftlichen Fachpersonal durchgeführt werden. Im Zuge dessen wäre es auch ratsam, die Limitationen von neurowissenschaftlichen Befunden stärker zu betonen. Immerhin legen Studien nahe, dass wissenschaftliche Befunde generell eher geglaubt werden, wenn sie neurowissenschaftlich begründet werden (McCabe und Castel 2008; Weisberg et al. 2009).

Während der Glaube an Lerntypen eindeutig der weiteste verbreitete Neuromythos war, sollten unserer Ansicht nach andere Neuromythen zuerst aufgelöst werden. Diese Priorisierung ist dadurch zu begründen, dass die praktische Umsetzung mancher Neuromythen vermutlich schädlicher ist als die Umsetzung anderer Neuromythen. Der Mythos von Lerntypen könnte dazu führen, dass Lehrkräfte Lernmaterial multimodal (für verschiedene Sinneskanäle) aufbereiten. Diese multimodale Aufbereitung dürfte lernförderlich sein (z. B. Van Someren et al. 1998), selbst wenn sie aus den falschen Gründen gemacht wird. Andere Studien zeigen auf, dass Lehrende Lerntypen umsetzen, weil sie Methodenvielfalt anstreben (Newton und Miah 2017).

Die Umsetzung anderer Neuromythen in der schulischen Praxis kann aus unserer Sicht allerdings zu schädlichen Praktiken führen. Zum Beispiel zeigte sich in der vorliegenden Studie eine verhältnismäßig hohe Zustimmungsrate $(51 \%)$ zum Mythos, dass nach kritischen Phasen in der Kindheit bestimmte Dinge nicht mehr gelernt werden können. Wenn Lehrkräfte diesen Neuromythos vertreten, kann es dazu führen, dass Kindern und Jugendlichen die Bildbarkeit in bestimmten Bereichen fälschlicherweise abgesprochen wird. Ebenso vertrat ein hoher Anteil der Lehramtsstudierenden (Zustimmungsrate 35\%) die Annahme, dass die Muttersprache vor allen anderen Sprachen erworben werden müsse, um Sprachen vollständig erlenen zu können. Eine Folge davon wäre, dass Lehrkräfte sich gegen das frühzeitige Erlernen von mehreren Sprachen aussprechen. Diese und andere Haltungen könnten 
sich fundamental auf den Lernprozess und die Entwicklung von Kindern und Jugendlichen auswirken. Studien zeigen auch auf, dass es diese Neuromythen sind, die zwischen guten und weniger guten Lehrkräften differenzieren (Horvath et al. 2018). Eine kritische Auseinandersetzung, wie man diesem Trend begegnet, ist daher von zunehmender gesellschaftlicher Relevanz.

\subsection{Faktorstruktur von Neuromythen und Neurofakten}

Mit der Ausnahme von Macdonald et al. (2017) nahmen Studien zu Neuromythen und Neurofakten a priori an, dass Neuromythen und Neurofakten jeweils ein Konstrukt abbilden, ohne diese Annahme zu überprüfen (vgl. Dekker et al. 2012; Ferrero et al. 2016; Howard-Jones et al. 2009; Papadatou-Pastou et al. 2017). Unsere Ergebnisse zeigen deutlich, dass den Neuromythen kein gemeinsamer Faktor zugrunde lag (konträr zu Hypothese 1.1), und dass nur 11 der 20 Neurofakten einen gemeinsamen Faktor bildeten (konträr zu Hypothese 1.2). Unsere Ergebnisse bestätigen damit die Forderung von Macdonald et al., dass die Faktorstruktur bei Studien zu Neuromythen und Neurofakten berücksichtigt werden sollte. Wenn keine solche eindimensionale Faktorstruktur gegeben ist, ist die Bildung von Gesamtwerten für weitere Berechnungen nicht zulässig (Wright und Stone 1979). Diese Ergebnisse zeigen auf, dass bisherige Befunde basierend auf aggregierten Neuromythen und Neurofakten kritisch hinterfragt werden müssen.

Macdonald et al. (2017) fanden für Teile ihrer Neuromythen einen Faktor, aber keinen für Neurofakten. In der vorliegenden Studie zeigte sich Umgekehrtes. Es konnte kein gemeinsamer Faktor für Neuromythen identifiziert werden, aber ein Faktor für Neurofakten. Dieses Ergebnis könnte aus mehreren Gründen zustande gekommen sein. Erstens analysierten Macdonald et al. die faktorielle Struktur rein explorativ, obwohl eindeutige Hypothesen zu der Struktur ableitbar sind. Zweitens setzte sich die Stichprobe bei Macdonald et al. aus drei Teilstichproben zusammen: Allgemeinbevölkerung, Lehrkräfte und neurowissenschaftliche Expertinnen und Experten. Die nicht interpretierbare Faktorstruktur für Neurofakten könnte damit erklärt werden, dass in diesen Teilstichproben verschiedene Faktorstrukturen vorhanden waren. Zum Beispiel sollten neurowissenschaftliche Expertinnen und Experten ein grundlegend anderes Verständnis haben, sodass hier Neurofakten möglicherweise etwas anderes messen als in den beiden anderen Teilstichproben. Drittens könnten Unterschiede auch durch das verwendete Antwortformat entstanden sein. Macdonald et al. gaben keine wei $\beta$ nicht Antwortkategorie vor, wodurch es erzwungen wurde, sich bei jeder Aussage zwischen wahr und falsch zu entscheiden. Betrachtet man in Tab. 2 die Häufigkeiten der weiß nicht Antwortkategorie in der vorliegenden Studie, zeigt sich, dass diese für manche Aussagen sehr häufig gewählt wurde (bis zu 46\%). Schließlich können die Unterschiede in den Faktorstrukturen auch darauf hinweisen, dass Neuromythen und Neurofakten trotz ihrer teilweise vergleichbaren Prävalenzen über Länder hinweg, länderspezifische Komponenten aufweisen. Dies würde bedeuten, dass es hinterfragt werden sollte, Neuromythen als ein länderübergreifendes Konstrukt zu behandeln. Auch hier scheint eine weitere Auseinandersetzung notwendig, um möglichen länderspezifischen Effekten in der Erhaltung und Vorbereitung von Neuromythen entgegenzuwirken. 
Es könnte noch weitere Ursachen dafür geben, dass wir keine Faktorstruktur für Neuromythen unter österreichischen Lehramtsstudierenden am Studienbeginn fanden. Erstens könnten Neuromythen getrennt voneinander auftreten. Es wäre denkbar, dass der Glaube an z. B. Lerntypen so stark verankert ist, dass er unabhängig vom Glauben an andere Neuromythen besteht. Künftige Studien könnten untersuchen, ob es grundlegende Neuromythen gibt, die unabhängig voneinander Zustimmung finden. Zweitens könnte es auch sein, dass verschiedene Neuromythen aufeinander aufbauen. Wenn man an ,... voneinander unabhängige Intelligenzen, lokalisiert in unterschiedlichen Gehirnregionen ..." glaubt, könnte dies dazu beitragen an Lerntypen zu glauben. Es sollte daher auch untersucht werden, ob es Neuromythen gibt, die einander bedingen. Drittens könnte es sein, dass verschiedene Neuromythen verschiedene Ursachen haben. Der Glaube an Lerntypen könnte aus eigener Schulerfahrung stammen, während ein Missverstehen von neurowissenschaftlichen Studien den Glauben an eine getrennte neuronale Verarbeitung in den Gehirnhälften fördert. Weitere Studien könnten daher auch gezielt die Entstehung von Neuromythen identifizieren. Viertens wäre es denkbar, dass es Subpopulationen gibt, wo bestimmte Neuromythen gemeinsam auftreten, oder sich gegenseitig ausschließen. Diese Annahmen könnten für österreichische Lehramtsstudierende mit den vorliegenden Daten überprüft werden. Interessierte Forscherinnen und Forscher seien hier auf unsere offengelegten Daten verwiesen unter https://osf.io/5tsfv/ (Krammer et al. 2019). Viertens könnte die Trennung zwischen Neuromythen und Neurofakten hinterfragt werden. Künftige Studien könnten demnach thematisieren, ab wann falsche Neurofakten zu Neuromythen werden. Schlussendlich könnten die genannten Überlegungen und die von uns nicht gefundene Faktorstruktur auch dafür sprechen, dass Neuromythen besser als formative Messmodelle verstanden werden sollten (für eine Übersicht s. Bollen und Bauldry 2011). Künftige Studien könnten daher neben Neuromythen gezielt Auswirkungen von Neuromythen untersuchen, und diese Annahmen in z. B. MIMIC-Modellen überprüfen.

\subsection{Wie hängen Neuromythen mit Neurofakten zusammen?}

Bisherige Studien haben berichtet, dass das Wissen zu Neurofakten mit dem Fehlglauben an Neuromythen zusammenhängt. Die Ergebnisse zeigten allerdings sowohl positive (Dekker et al. 2012; Ferrero et al. 2016) als auch negative (Howard-Jones et al. 2009; Papadatou-Pastou et al. 2017) Assoziationen. Leider haben bisherige Studien diese Zusammenhänge analysiert, ohne die Faktorstruktur von Neuromythen und Neurofakten zu berücksichtigen. Die vorliegende Studie ist die erste, die unter Berücksichtigung der Faktorstruktur das Wissen zu Neurofakten mit Neuromythen in Zusammenhang setzt. Wurde die Faktorstruktur berücksichtigt, zeigte sich, dass für die meisten Neuromythen deren Ablehnung bzw. Zustimmung nicht mit dem Wissen zu Neurofakten zusammenhing (konträr zu Hypothese 2). Nur bei einem Neuromythos ging dessen korrekte Ablehnung mit einem höheren Wissen einher als dessen fälschliche Zustimmung. Dieses Ergebnismuster spricht dafür, dass Neurofakten und Neuromythen weitgehend unabhängig voneinander sind. Für die Ausbildung von Lehrkräften implizieren diese Ergebnisse, dass nicht nur 
neurowissenschaftliches Wissen vermittelt, sondern gezielt an der Auflösung von Neuromythen gearbeitet werden sollte.

Wenn die empirische Faktorstruktur von Neuromythen und Neurofakten wie in vorherigen Studien unberücksichtigt bleibt, zeigten sich Zusammenhänge zwischen Neuromythen und Neurofakten. Dies legt nahe, dass bisherige Ergebnisse (s. Dekker et al. 2012; Ferrero et al. 2016; Howard-Jones et al. 2009; Papadatou-Pastou et al. 2017) methodische Artefakte sein könnten. Dies wird auch durch das Zusatzergebnis bestärkt, wonach ein größeres Wissen über das Gehirn (mehr Neurofakten richtig identifiziert) sowohl mit einer stärkeren Annahme (fälschlicherweise geglaubt) als auch stärkeren Ablehnung (korrekterweise abgelehnt) von Neuromythen einherging (siehe Tab. 5, Anhang). Dieser augenscheinlich widersprüchliche Befund lässt sich durch Berücksichtigung der dritten Antwortkategorie - weiß nicht - erklären. Durch diese Antwortkategorie sind Ergebnisse zu der korrekten Ablehnung von Neuromythen nicht mehr komplementär zu deren falscher Zustimmung. In keiner der bisherigen Studien wurden beide Zusammenhänge berichtet.

\subsection{Limitationen und Konklusion}

Trotz der verglichen mit bisherigen Studien großen Stichprobe bleibt offen, ob sich unsere Befunde auf Lehramtsstudierende in ganz Österreich generalisieren lassen. Die vorliegende Studie basiert auf Lehramtsstudierenden in einem Bundesland.

Eine weitere offene Frage bezieht sich auf die Einbettung von Neuromythen in der österreichischen Schulpraxis. Bislang wurde dies nur in einer Studie (Simmonds 2014) mittels Fragebogen bei Lehrpersonen in Großbritannien erhoben. In dieser zeigte sich beispielsweise, dass der Mythos der Lerntypen bei einem Großteil der Lehrkräften Eingang in die Unterrichtspraxis fand und dass dieser institutionell (von der Schule) vermittelt wurde. In zukünftigen Studien sollte auch im österreichischen Bildungssystem untersucht werden, in welchem Ausmaß Neuromythen in der Aus-, Fort- und Weiterbildung abgebaut oder sogar verstärkt werden und inwieweit Neuromythen tatsächlich Einfluss auf die Unterrichtspraxis haben. Hierfür wäre es erforderlich im Längsschnittdesign Lehramtsstudierende, Lehrkräfte aber auch Lehrerbildnerinnen und Lehrerbildner zu befragen.

Trotz dieser Limitationen ist die vorliegende Studie die erste, die die Prävalenz von Neuromythen im österreichischen Bildungssystem zeigt. Sie ist auch die erste, die zeigt, dass Neuromythen und Neurofakten nur zusammenhängen, wenn vorhandene Faktorstrukturen unberücksichtigt bleiben. Wurde die Faktorstruktur berücksichtigt, zeigte sich kein Zusammenhang.

Schlussendlich ist eine detaillierte Auseinandersetzung mit der Entstehung und Aufrechterhaltung von Neuromythen im Bildungssystem von unmittelbarer Relevanz für eine evidenzbasierte Schulpraxis sowie für die Aus-, Fort- und Weiterbildung unserer Lehrkräfte. Um dem Bestehen von Neuromythen entgegen zu wirken, sollte die Lehramtsausbildung nicht allgemein mehr neurowissenschaftliche Befunde vermitteln, sondern gezielt auf Neuromythen eingehen. Im Fokus sollten hier jene Mythen stehen, deren Umsetzung negative Konsequenzen für die schulische Praxis haben könnte. 
Funding Open access funding provided by University of Graz.

\section{Anhang}

Tab. 3 Relative Häufigkeiten der gewählten Antwortkategorien (richtig, falsch, weiß nicht) der vorgegebenen Neuromythen und Neurofakten, aufgegliedert für die beiden Teilstichproben

\begin{tabular}{|c|c|c|c|c|c|c|c|c|}
\hline & \multicolumn{4}{|c|}{$\begin{array}{l}\text { Teilstichprobe } 1 \\
(n=255)\end{array}$} & \multicolumn{4}{|c|}{$\begin{array}{l}\text { Teilstichprobe } 2 \\
(n=327)\end{array}$} \\
\hline & $\mathrm{R}$ & $\mathrm{F}$ & WN & Miss & $\mathrm{R}$ & $\mathrm{F}$ & WN & Miss \\
\hline $\begin{array}{l}\text { Kinder müssen ihre Muttersprache } \\
\text { (L1) erwerben, bevor sie weitere } \\
\text { Sprachen (L2, L3) lernen. Ist } \\
\text { dies nicht der Fall, wird keine der } \\
\text { Sprachen vollständig erworben. } \\
\text { (Quelle: Dekker et al. 2012) }\end{array}$ & 0,35 & 0,51 & 0,14 & 0,01 & 0,35 & 0,55 & 0,10 & 0,00 \\
\hline $\begin{array}{l}\text { Sobald Schüler nicht genug Was- } \\
\text { ser (=6-8 Gläser pro Tag) zu sich } \\
\text { nehmen, schrumpft das Gehirn. } \\
\text { (Quelle: Dekker et al. 2012) }\end{array}$ & 0,07 & 0,72 & 0,20 & 0,00 & 0,02 & 0,76 & 0,21 & 0,00 \\
\hline $\begin{array}{l}\text { Es ist wissenschaftlich erwie- } \\
\text { sen, dass Fettsäure (Omega-3, } \\
\text { Omega-6) enthaltende Nahrungs- } \\
\text { ergänzungsmittel eine positive } \\
\text { Auswirkung auf den akademischen } \\
\text { Erfolg haben. (Quelle: Dekker } \\
\text { et al. 2012) }\end{array}$ & 0,36 & 0,18 & 0,46 & 0,00 & 0,40 & 0,14 & 0,46 & 0,00 \\
\hline $\begin{array}{l}\text { Wir nutzen nur } 10 \% \text { unseres Ge- } \\
\text { hirns. (Quelle: Dekker et al. 2012) }\end{array}$ & 0,45 & 0,36 & 0,18 & 0,01 & 0,42 & 0,38 & 0,19 & 0,00 \\
\hline $\begin{array}{l}\text { Unterschiede in der Gehirndo- } \\
\text { minanz (linke oder rechte Ge- } \\
\text { hirnhälfte) können individuelle } \\
\text { Unterschiede zwischen Lernenden } \\
\text { erklären. (Quelle: Dekker et al. } \\
\text { 2012) }\end{array}$ & 0,79 & 0,04 & 0,17 & 0,00 & 0,84 & 0,02 & 0,13 & 0,00 \\
\hline $\begin{array}{l}\text { Die Gehirne von Jungen und } \\
\text { Mädchen entwickeln sich gleich } \\
\text { schnell. (Quelle: Dekker et al. } \\
\text { 2012) }\end{array}$ & 0,18 & 0,55 & 0,25 & 0,01 & 0,20 & 0,53 & 0,26 & 0,01 \\
\hline $\begin{array}{l}\text { Die Gehirnentwicklung ist zwi- } \\
\text { schen dem 11.-12. Lebensjahr } \\
\text { abgeschlossen. (Quelle: Dekker } \\
\text { et al. 2012) }\end{array}$ & 0,04 & 0,65 & 0,29 & 0,02 & 0,03 & 0,67 & 0,30 & 0,00 \\
\hline $\begin{array}{l}\text { In der Kindheit gibt es kritische } \\
\text { Phasen, nach denen bestimmte } \\
\text { Dinge nicht mehr gelernt werden } \\
\text { können. (Quelle: Dekker et al. } \\
\text { 2012) }\end{array}$ & 0,51 & 0,30 & 0,19 & 0,00 & 0,51 & 0,28 & 0,21 & 0,00 \\
\hline $\begin{array}{l}\text { Schüler lernen besser, wenn ihnen } \\
\text { Informationen entsprechend ihrem } \\
\text { Lerntyp (z. B. visuell, auditiv, } \\
\text { kinästhetisch) vermittelt werden. } \\
\text { (Quelle: Dekker et al. 2012) }\end{array}$ & 0,96 & 0,01 & 0,02 & 0,00 & 0,97 & 0,00 & 0,02 & 0,00 \\
\hline
\end{tabular}


Tab. 3 (Fortsetzung)

\begin{tabular}{|c|c|c|c|c|c|c|c|c|}
\hline & \multicolumn{4}{|c|}{$\begin{array}{l}\text { Teilstichprobe } 1 \\
(n=255)\end{array}$} & \multicolumn{4}{|c|}{$\begin{array}{l}\text { Teilstichprobe } 2 \\
(n=327)\end{array}$} \\
\hline & $\mathrm{R}$ & $\mathrm{F}$ & WN & Miss & $\mathrm{R}$ & $\mathrm{F}$ & WN & Miss \\
\hline $\begin{array}{l}\text { Reizreiche Umgebungen verbes- } \\
\text { sern die Gehirnentwicklung von } \\
\text { Kindergartenkindern. (Quelle: } \\
\text { Dekker et al. 2012) }\end{array}$ & 0,60 & 0,13 & 0,27 & 0,00 & 0,55 & 0,19 & 0,26 & 0,00 \\
\hline $\begin{array}{l}\text { Kinder sind weniger aufnahme- } \\
\text { fähig nachdem sie zuckerhaltige } \\
\text { Getränke und/oder Snacks zu sich } \\
\text { genommen haben. (Quelle: Dekker } \\
\text { et al. 2012) }\end{array}$ & 0,41 & 0,31 & 0,28 & 0,00 & 0,38 & 0,37 & 0,24 & 0,00 \\
\hline $\begin{array}{l}\text { Übungen der Körper-Augen-Ko- } \\
\text { ordination können sich positiv } \\
\text { auf die Lesefähigkeit auswirken. } \\
\text { (Quelle: Dekker et al. 2012) }\end{array}$ & 0,81 & 0,04 & 0,15 & 0,00 & 0,79 & 0,04 & 0,17 & 0,00 \\
\hline $\begin{array}{l}\text { Intelligenz wird vererbt und kann } \\
\text { nicht durch Erfahrung oder Um- } \\
\text { welteinflüsse verändert werden. } \\
\text { (Quelle: Stellungnahme zur Früh- } \\
\text { kindlichen Sozialisation 2014) }\end{array}$ & 0,07 & 0,82 & 0,10 & 0,00 & 0,05 & 0,88 & 0,06 & 0,00 \\
\hline $\begin{array}{l}\text { Lernschwierigkeiten, die in Ver- } \\
\text { bindung mit entwicklungsbeding- } \\
\text { ten Unterschieden in der Gehirn- } \\
\text { funktion stehen, können nicht } \\
\text { durch Bildung korrigiert werden. } \\
\text { (Quelle: Dekker et al. 2012) }\end{array}$ & 0,18 & 0,44 & 0,38 & 0,00 & 0,20 & 0,52 & 0,28 & 0,00 \\
\hline $\begin{array}{l}\text { Kurzzeitige Koordinationsübungen } \\
\text { können die Integration zwischen } \\
\text { linker und rechter Gehirnhälfte } \\
\text { verbessern }\end{array}$ & 0,85 & 0,06 & 0,09 & 0,00 & 0,89 & 0,04 & 0,07 & 0,00 \\
\hline $\begin{array}{l}\text { Wenn wir schlafen ist das Gehirn } \\
\text { nicht aktiv. (Quelle: Dekker et al. } \\
\text { 2012) }\end{array}$ & 0,01 & 0,93 & 0,05 & 0,00 & 0,01 & 0,96 & 0,03 & 0,00 \\
\hline $\begin{array}{l}\text { Es gibt nicht nur eine, sondern } \\
\text { mehrere, voneinander unabhängige } \\
\text { Intelligenzen, die in unterschied- } \\
\text { lichen Gehirnregionen lokalisiert } \\
\text { sind. (Quelle: Stellungnahme } \\
\text { zur Frühkindlichen Sozialisation } \\
\text { 2014) }\end{array}$ & 0,56 & 0,05 & 0,38 & 0,00 & 0,63 & 0,09 & 0,28 & 0,00 \\
\hline $\begin{array}{l}\text { Der Unterricht sollte so gestaltet } \\
\text { werden, dass beide Gehirnhälften } \\
\text { beansprucht werden. (Quelle: } \\
\text { OECD 2002) }\end{array}$ & 0,84 & 0,02 & 0,14 & 0,00 & 0,88 & 0,02 & 0,10 & 0,00 \\
\hline $\begin{array}{l}\text { Der mehrjährige Schulbesuch } \\
\text { macht die Kinder weniger kreativ. } \\
\text { Am kreativsten sind Kinder vor } \\
\text { der Einschulung. (Quelle: Wagen- } \\
\text { hofer et al. 2013) }\end{array}$ & 0,42 & 0,30 & 0,27 & 0,00 & 0,42 & 0,33 & 0,25 & 0,00 \\
\hline
\end{tabular}


Tab. 3 (Fortsetzung)

\begin{tabular}{|c|c|c|c|c|c|c|c|c|}
\hline & \multicolumn{4}{|c|}{$\begin{array}{l}\text { Teilstichprobe } 1 \\
(n=255)\end{array}$} & \multicolumn{4}{|c|}{$\begin{array}{l}\text { Teilstichprobe } 2 \\
(n=327)\end{array}$} \\
\hline & $\mathrm{R}$ & $\mathrm{F}$ & WN & Miss & $\mathrm{R}$ & $\mathrm{F}$ & WN & Miss \\
\hline $\begin{array}{l}\text { Hochbegabte brauchen nicht zu } \\
\text { lernen, um gute schulische Leis- } \\
\text { tungen zu erbringen. (Quelle: } \\
\text { Stern und Neubauer 2013, S. 181) }\end{array}$ & 0,07 & 0,82 & 0,11 & 0,00 & 0,04 & 0,90 & 0,06 & 0,00 \\
\hline $\begin{array}{l}\text { Wir nutzen unser Gehirn 24h am } \\
\text { Tag. (Quelle: Dekker et al. 2012) }\end{array}$ & 0,84 & 0,12 & 0,04 & 0,00 & 0,81 & 0,15 & 0,03 & 0,00 \\
\hline $\begin{array}{l}\text { Das Gehirn von Jungs ist größer } \\
\text { als das Gehirn von Mädchen. } \\
\text { (Quelle: Dekker et al. 2012) }\end{array}$ & 0,19 & 0,61 & 0,20 & 0,00 & 0,17 & 0,65 & 0,17 & 0,01 \\
\hline $\begin{array}{l}\text { Die Funktionen von beschädigten } \\
\text { Gehirnregionen können von ande- } \\
\text { ren Gehirnregionen übernommen } \\
\text { werden. (Quelle: Dekker et al. } \\
\text { 2012) }\end{array}$ & 0,51 & 0,24 & 0,24 & 0,00 & 0,54 & 0,28 & 0,17 & 0,00 \\
\hline $\begin{array}{l}\text { Die linke und rechte Gehirnhälfte } \\
\text { arbeiten immer zusammen. (Quel- } \\
\text { le: Dekker et al. 2012) }\end{array}$ & 0,36 & 0,51 & 0,13 & 0,00 & 0,25 & 0,59 & 0,15 & 0,00 \\
\hline $\begin{array}{l}\text { Information ist in einem Netzwerk } \\
\text { von Nervenzellen, das über das } \\
\text { Gehirn verteilt ist gespeichert. } \\
\text { (Quelle: Dekker et al. 2012) }\end{array}$ & 0,87 & 0,03 & 0,09 & 0,01 & 0,84 & 0,05 & 0,10 & 0,01 \\
\hline $\begin{array}{l}\text { Durch Verhaltensinterventionen } \\
\text { lassen sich ungewöhnliche Ent- } \\
\text { wicklungsverläufe verändern. } \\
\text { (Quelle: Dekker et al. 2012) }\end{array}$ & 0,59 & 0,07 & 0,33 & 0,00 & 0,63 & 0,05 & 0,32 & 0,00 \\
\hline $\begin{array}{l}\text { Lernen erfolgt nicht durch die Bil- } \\
\text { dung neuer Nervenzellen. (Quelle: } \\
\text { Dekker et al. 2012) }\end{array}$ & 0,36 & 0,35 & 0,28 & 0,00 & 0,40 & 0,35 & 0,24 & 0,01 \\
\hline $\begin{array}{l}\text { Das Auslassen des Frühstücks } \\
\text { kann den akademischen Erfolg } \\
\text { beeinflussen. (Quelle: Dekker et al. } \\
\text { 2012) }\end{array}$ & 0,42 & 0,36 & 0,22 & 0,00 & 0,43 & 0,33 & 0,23 & 0,00 \\
\hline $\begin{array}{l}\text { Gehirnverletzungen im frühen } \\
\text { Kindesalter haben vergleichswei- } \\
\text { se negativere Folgen, als solche } \\
\text { im Vorschulalter. (Quelle: Stel- } \\
\text { lungnahme zur Frühkindlichen } \\
\text { Sozialisation 2014) }\end{array}$ & 0,36 & 0,19 & 0,45 & 0,00 & 0,40 & 0,19 & 0,40 & 0,00 \\
\hline $\begin{array}{l}\text { Lernen geschieht im Gehirn durch } \\
\text { die Veränderung neuronaler Ver- } \\
\text { bindungen. (Quelle: Dekker et al. } \\
\text { 2012) }\end{array}$ & 0,66 & 0,05 & 0,27 & 0,02 & 0,73 & 0,05 & 0,21 & 0,01 \\
\hline $\begin{array}{l}\text { Lernfähigkeit besteht in einigen } \\
\text { Funktionsbereichen ein Leben } \\
\text { lang. (Quelle: Stellungnahme } \\
\text { zur Frühkindlichen Sozialisation } \\
\text { 2014) }\end{array}$ & 0,91 & 0,03 & 0,05 & 0,00 & 0,90 & 0,04 & 0,06 & 0,00 \\
\hline
\end{tabular}


Tab. 3 (Fortsetzung)

\begin{tabular}{|c|c|c|c|c|c|c|c|c|}
\hline & \multicolumn{4}{|c|}{$\begin{array}{l}\text { Teilstichprobe } 1 \\
(n=255)\end{array}$} & \multicolumn{4}{|c|}{$\begin{array}{l}\text { Teilstichprobe } 2 \\
(n=327)\end{array}$} \\
\hline & $\mathrm{R}$ & $\mathrm{F}$ & WN & Miss & $\mathrm{R}$ & F & WN & Miss \\
\hline $\begin{array}{l}\text { Die normale Entwicklung des Ge- } \\
\text { hirns bedingt sowohl die Bildung } \\
\text { als auch das Absterben von Ge- } \\
\text { hirnzellen. (Quelle: Dekker et al. } \\
\text { 2012) }\end{array}$ & 0,70 & 0,07 & 0,23 & 0,00 & 0,72 & 0,05 & 0,22 & 0,00 \\
\hline $\begin{array}{l}\text { Anstrengende Bewegung kann } \\
\text { die geistige Leistungsfähigkeit } \\
\text { fördern. (Quelle: Dekker et al. } \\
\text { 2012) }\end{array}$ & 0,67 & 0,09 & 0,24 & 0,00 & 0,68 & 0,12 & 0,19 & 0,00 \\
\hline $\begin{array}{l}\text { Der zirkadiane Rhythmus „Schlaf- } \\
\text { Wach-Rhythmus“ verändert sich } \\
\text { während der Pubertät, was dazu } \\
\text { führt, dass SchülerInnen während } \\
\text { der ersten Stunden des Schultages } \\
\text { müde sind. (Quelle: Dekker et al. } \\
\text { 2012) }\end{array}$ & 0,58 & 0,15 & 0,27 & 0,00 & 0,59 & 0,14 & 0,27 & 0,00 \\
\hline $\begin{array}{l}\text { Regelmäßiger Koffeinkonsum } \\
\text { verringert die Wachsamkeit/ } \\
\text { Aufmerksamkeit. (Quelle: Dek- } \\
\text { ker et al. 2012) }\end{array}$ & 0,35 & 0,31 & 0,35 & 0,00 & 0,37 & 0,28 & 0,34 & 0,01 \\
\hline $\begin{array}{l}\text { Wiederholtes und langfristiges } \\
\text { Training bestimmter geistiger Ab- } \\
\text { läufe kann die Form und Struktur } \\
\text { mancher Gehirnregionen verän- } \\
\text { dern. (Quelle: Dekker et al. 2012) }\end{array}$ & 0,83 & 0,05 & 0,12 & 0,00 & 0,84 & 0,06 & 0,10 & 0,00 \\
\hline $\begin{array}{l}\text { Lern- und Gedächtnisleistungen } \\
\text { können mit steigendem Inhalts- } \\
\text { wissen erhöht werden. (Quelle: } \\
\text { Stellungnahme zur Frühkindlichen } \\
\text { Sozialisation 2014) }\end{array}$ & 0,81 & 0,04 & 0,13 & 0,01 & 0,80 & 0,07 & 0,13 & 0,00 \\
\hline $\begin{array}{l}\text { Die Entstehung neuer Gehirnver- } \\
\text { bindungen ist bis ins hohe Alter } \\
\text { möglich. (Quelle: Dekker et al. } \\
\text { 2012) }\end{array}$ & 0,87 & 0,03 & 0,09 & 0,00 & 0,83 & 0,05 & 0,11 & 0,01 \\
\hline $\begin{array}{l}\text { In der Kindheit gibt es sensible } \\
\text { Phasen in denen es einfacher ist } \\
\text { Dinge zu lernen. (Quelle: Dekker } \\
\text { et al. 2012) }\end{array}$ & 0,92 & 0,02 & 0,06 & 0,00 & 0,92 & 0,01 & 0,07 & 0,00 \\
\hline $\begin{array}{l}\text { Existierende Synapsen werden } \\
\text { durch Erfahrungen entweder ver- } \\
\text { stärkt oder eliminiert. (Quelle: } \\
\text { Stellungnahme zur Frühkindlichen } \\
\text { Sozialisation 2014) }\end{array}$ & 0,49 & 0,11 & 0,38 & 0,01 & 0,55 & 0,14 & 0,30 & 0,01 \\
\hline
\end{tabular}

Die relative Häufigkeit der korrekten Antwortkategorie ist kursiv gesetzt

$R$ richtig, $F$ falsch, $W N$ weiß nicht, miss fehlende Werte 


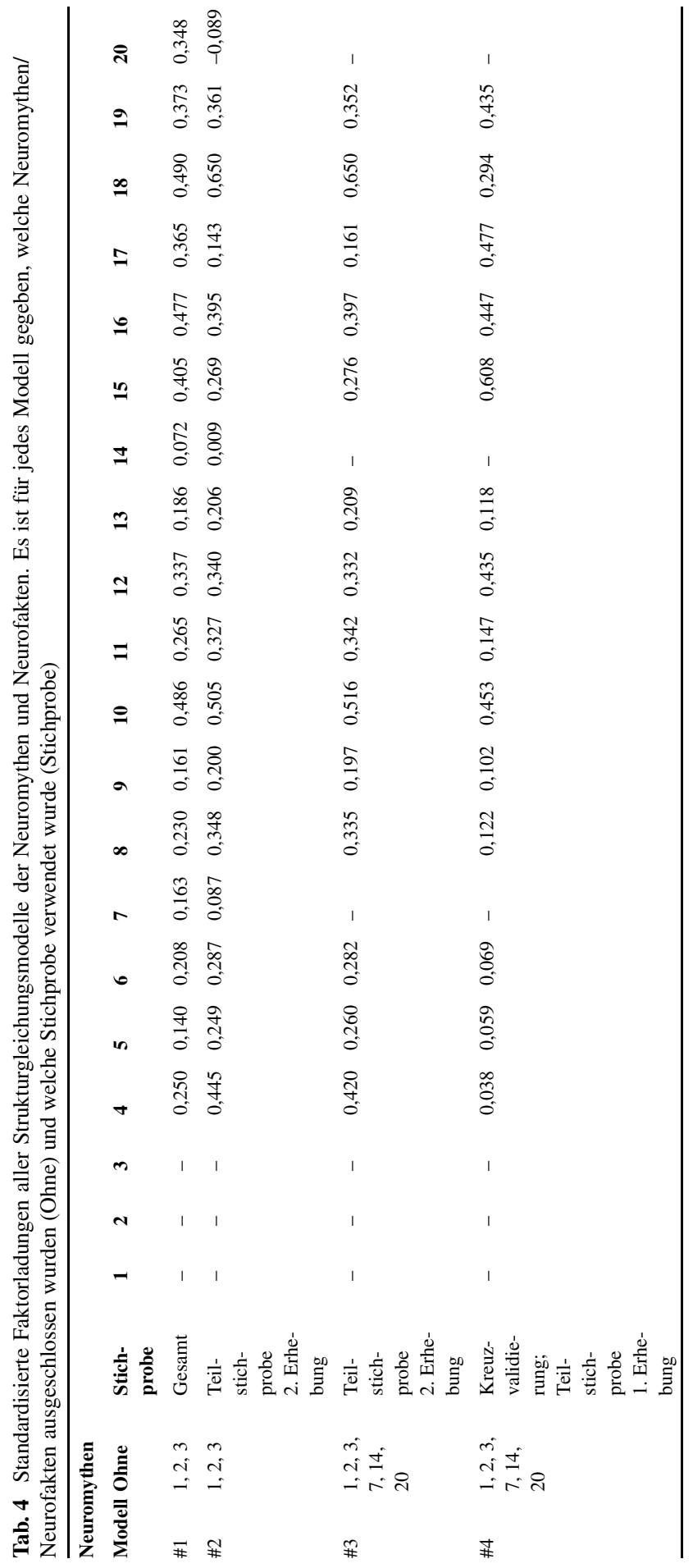




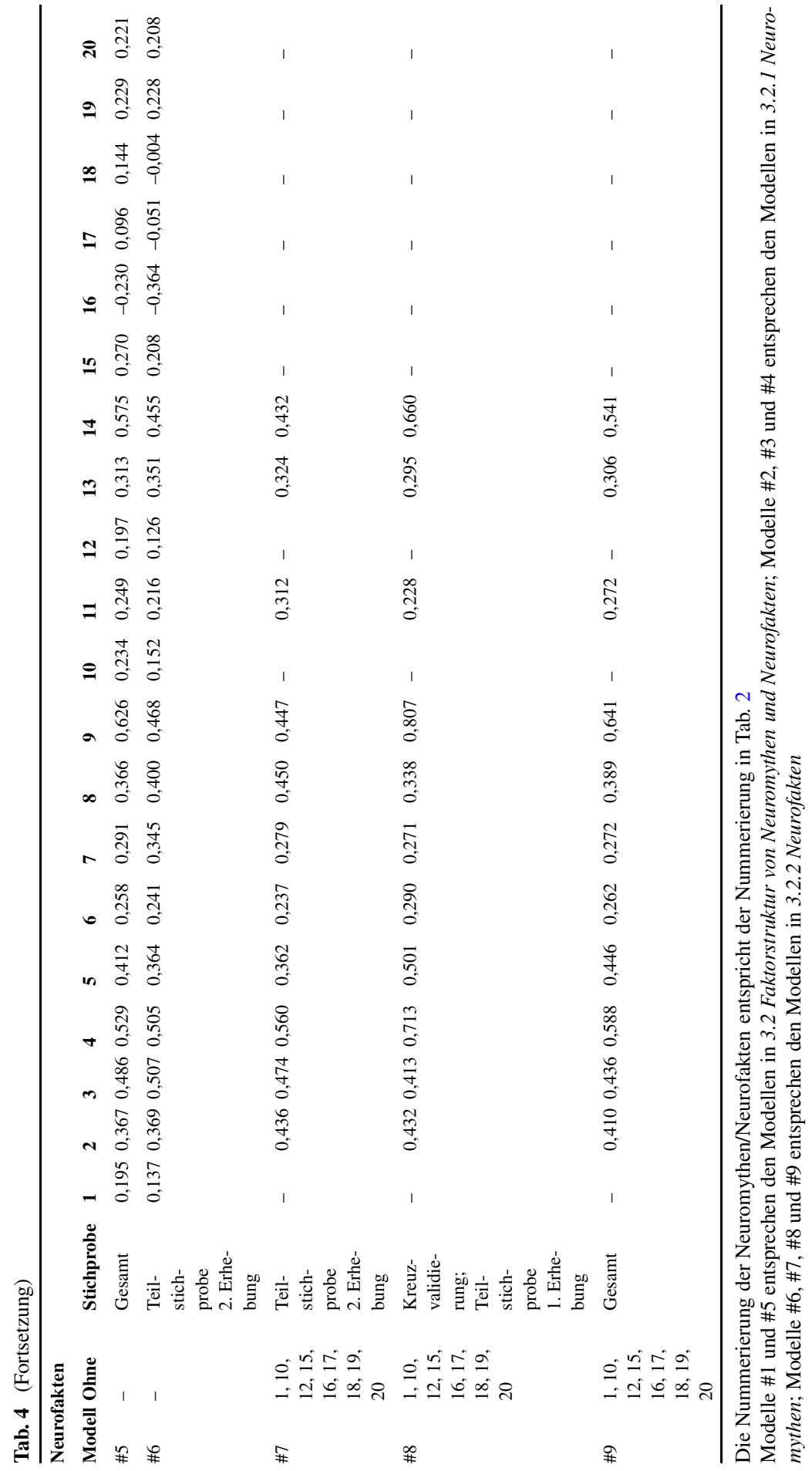


Tab. 5 Korrelationen der Häufigkeit der gewählten Antwortkategorien (richtig, falsch, oder weiß nicht) der vorgegebenen Neuromythen und Neurofakten. Hierfür wurde keine Faktorstruktur berücksichtigt

\begin{tabular}{llllllll}
\hline & \multicolumn{3}{c}{ Neuromythen } & \multicolumn{4}{c}{ Neurofakten } \\
& & R & F & WN & R & F & WN \\
\hline Neuromythen & R & - & $-0,240$ & $-0,627$ & 0,314 & 0,112 & $-0,362$ \\
& F & - & - & $-0,600$ & 0,215 & 0,286 & $-0,394$ \\
\multirow{5}{*}{ Neurofakten } & WN & - & - & - & $-0,426$ & $-0,319$ & 0,609 \\
& R & - & - & - & - & $-0,250$ & $-0,751$ \\
& F & - & - & - & - & - & $-0,445$ \\
& WN & - & - & - & - & - & - \\
\hline
\end{tabular}

Bei einer Stichprobengröße von 582 ist eine Korrelation ab $\pm 0,081$ signifikant $(p<0,05)$

$R$ richtig, $F$ falsch, $W N$ weiß nicht

Open Access Dieser Artikel wird unter der Creative Commons Namensnennung 4.0 International Lizenz (http://creativecommons.org/licenses/by/4.0/deed.de) veröffentlicht, welche die Nutzung, Vervielfältigung, Bearbeitung, Verbreitung und Wiedergabe in jeglichem Medium und Format erlaubt, sofern Sie den/die ursprünglichen Autor(en) und die Quelle ordnungsgemäß nennen, einen Link zur Creative Commons Lizenz beifügen und angeben, ob Änderungen vorgenommen wurden.

\section{Literatur}

An, D., \& Carr, M. (2017). Learning styles theory fails to explain learning and achievement: recommendations for alternative approaches. Personality and Individual Differences, 116, 410-416. https://doi. org/10.1016/j.paid.2017.04.050.

Bollen, K. A., \& Bauldry, S. (2011). Three cs in measurement models: causal indicators, composite indicators, and covariates. Psychological Methods, 16(3), 265-284. https://doi.org/10.1037/a0024448.

Bowers, J. S. (2016). Psychology, not educational neuroscience, is the way forward for improving educational outcomes for all children: reply to Gabrieli (2016) and Howard-jones et al. (2016). Psychological Review, 123(5), 628-635. https://doi.org/10.1037/rev0000043.

Dekker, S., Lee, N.C., Howard-Jones, P. A., \& Jolles, J. (2012). Neuromyths in education: prevalence and predictors of misconceptions among teachers. Frontiers in Psychology, 3, 1-8. https://doi.org/10. 3389/fpsyg.2012.00429.

Deligiannidi, K., \& Howard-Jones, P.A. (2015). The neuroscience literacy of teachers in Greece. Procedia-Social and Behavioral Sciences, 174, 3909-3915. https://doi.org/10.1016/j.sbspro.2015.01. 1133.

Dubinsky, J. M., Roehrig, G., \& Varma, S. (2013). Infusing neuroscience into teacher professional development. Educational Researcher, 42(6), 317-329. https://doi.org/10.3102/0013189X13499403.

Düvel, N., Wolf, A., \& Kopiez, R. (2017). Neuromyths in music education: prevalence and predictors of misconceptions among teachers and students. Frontiers in Psychology, 8, 1-12. https://doi.org/10. 3389/fpsyg.2017.00629.

Ferrero, M., Garaizar, P., \& Vadillo, M. A. (2016). Neuromyths in education: prevalence among Spanish teachers and an exploration of cross-cultural variation. Frontiers in Human Neuroscience, 10, 1-11. https://doi.org/10.3389/fnhum.2016.00496.

Greiff, S., \& Heene, M. (2017). Why psychological assessment needs to start worrying about model fit. European Journal of Psychological Assessment, 33(5), 313-317. https://doi.org/10.1027/1015-5759/ a000450.

Hardiman, M., Rinne, L., Gregory, E., \& Yarmolinskaya, J. (2012). Neuroethics, neuroeducation, and classroom teaching: where the brain sciences meet pedagogy. Neuroethics, 5(2), 135-143. https:// doi.org/10.1007/s12152-011-9116-6.

Harmes, M., Huijser, H., Danaher, P., \& Haq, M. U. (2015). Myths in education, learning and teaching: policies, practices and principles. Berlin Heidelberg: Springer.

Horvath, J.C., Donoghue, G. M., Horton, A. J., Lodge, J. M., \& Hattie, J. A. C. (2018). On the irrelevance of neuromyths to teacher effectiveness: Comparing neuro-literacy levels amongst award-winning and 
non-award winning teachers. Frontiers in Psychology, 9, 1-5. https://doi.org/10.3389/fpsyg.2018. 01666.

Howard-Jones, P. A. (2014). Neuroscience and education: Myths and messages. Nature Reviews Neuroscience, 15(12), 817-824. https://doi.org/10.1038/nrn3817.

Howard-Jones, P. A., \& Fenton, K. D. (2012). The need for interdisciplinary dialogue in developing ethical approaches to neuroeducational research. Neuroethics, 5(2), 119-134. https://doi.org/10.1007/ s12152-011-9101-0.

Howard-Jones, P.A., Franey, L., Mashmoushi, R., \& Liao, Y.-C. (2009). The neuroscience literacy of trainee teachers. In British Educational Research Association Annual Conference (S. 1-39).

Hu, L., \& Bentler, P. M. (1999). Cutoff criteria for fit indexes in covariance structure analysis: conventional criteria versus new alternatives. Structural Equation Modeling: A Multidisciplinary Journal, 6(1), 1-55. https://doi.org/10.1080/10705519909540118.

Im, S. H., Cho, J. Y., Dubinsky, J. M., \& Varma, S. (2018). Taking an educational psychology course improves neuroscience literacy but does not reduce belief in neuromyths. PLoS ONE, 13(2), 1-19. https:// doi.org/10.1371/journal.pone.0192163.

Karakus, O., Howard-Jones, P. A., \& Jay, T. (2015). Primary and secondary school teachers' knowledge and misconceptions about the brain in Turkey. Procedia-Social and Behavioral Sciences, 174, 1933-1940. https://doi.org/10.1016/j.sbspro.2015.01.858.

Krammer, G., Vogel, S., Yardimci, T., \& Grabner, R.H. (2019). Neuromythen im Lehramtsstudium/ Neuromyths in Teacher Education (Artikel/Paper). osf.io/5tsfv. Zugegriffen: 18. März 2019.

Lei, P.W. (2009). Evaluating estimation methods for ordinal data in structural equation modeling. Quality \& Quantity, 43(3), 495-507. https://doi.org/10.1007/s11135-007-9133-z.

Macdonald, K., Germine, L., Anderson, A., Christodoulou, J., \& McGrath, L. M. (2017). Dispelling the myth: Training in education or neuroscience decreases but does not eliminate beliefs in neuromyths. Frontiers in Psychology, 8, 1-16. https://doi.org/10.3389/fpsyg.2017.01314.

Marsh, H.W., Hau, K.-T., \& Wen, Z. (2004). In search of golden rules: comment on hypothesis-testing approaches to setting cutoff values for fit indexes and dangers in overgeneralizing $\mathrm{Hu}$ and Bentler's (1999) findings. Structural Equation Modeling, 11(3), 452-483. https://doi.org/10.1207/ s15328007sem1103.

McCabe, D. P., \& Castel, A. D. (2008). Seeing is believing: The effect of brain images on judgments of scientific reasoning. Cognition, 107(1), 343-352. https://doi.org/10.1016/j.cognition.2007.07.017.

Newton, P. M., \& Miah, M. (2017). Evidence-based higher education-Is the learning styles "myth" important? Frontiers in Psychology, 8, 1-9. https://doi.org/10.3389/fpsyg.2017.00444.

OECD (2002). Understanding the Brain: Towards a New Learning Science. Paris: OECD Publishing.

Papadatou-Pastou, M., Haliou, E., \& Vlachos, F. (2017). Brain knowledge and the prevalence of neuromyths among prospective teachers in Greece. Frontiers in Psychology, 8 https://doi.org/10.3389/ fpsyg.2017.00804.

Pashler, H., McDaniel, M., Rohrer, D., \& Bjork, R. (2008). Learning styles: concepts and evidence. Psychological Science in the Public Interest, 9, 105-119.

Pei, X., Howard-Jones, P. A., Zhang, S., Liu, X., \& Jin, Y. (2015). Teachers' understanding about the brain in east China. Procedia-Social and Behavioral Sciences, 174, 3681-3688. https://doi.org/10.1016/j. sbspro.2015.01.1091.

R Core Team (2018). R: a language and environment for statistical computing. Vienna: R Core Team. https://www.r-project.org/.

Rato, J.R., Abreu, A. M., \& Castro-Caldas, A. (2013). Neuromyths in education: what is fact and what is fiction for Portuguese teachers? Educational Research, 55(4), 441-453. https://doi.org/10.1080/ 00131881.2013 .844947$.

Rosseel, Y. (2012). lavaan: an R package for structural equation modelin. Journal of Statistical Software, 48(2), 1-36. https://doi.org/10.18637/jss.v048.i02.

Savalei, V., \& Rhemtulla, M. (2013). The performance of robust test statistics with categorical data. British Journal of Mathematical and Statistical Psychology, 66(2), 201-223. https://doi.org/10.1111/j.20448317.2012.02049.x.

Simmonds, A. (2014). How neuroscience is affecting education: Report of teacher and parent surveys. Education Research, Wellcome Trust, London, (January), 1-13. https://wellcome.ac.uk/sites/default/ files/wtp055240.pdf. Zugegriffen: 05. April 2019.

Stern, E., \& Neubauer, A.C. (2013). Intelligenz-Große Unterschiede und ihre Folgen. München: DVA.

Thomas, M.S.C. (2013). Educational neuroscience in the near and far future: predictions from the analogy with the history of medicine. Trends in Neuroscience and Education, 2(1), 23-26. https://doi.org/10. 1016/j.tine.2012.12.001. 
Van Someren, M.W., Reimann, P., \& Boshuizen, H. (1998). Learning with multiple representations. Advances in learning and instruction series. New York: Elsevier Science, Inc.

Wagenhofer, E., Kriechbaum, S., \& Stern, A. (2013). alphabet: Angst oder Liebe. Salzburg: Ecowin.

Weisberg, D.S., Keil, F.C., Goodstein, J., Rawson, E., \& Gray, R. (2009). NIH Public Access, 20(3), 470-477. https://doi.org/10.1162/jocn.2008.20040.The.

Westermann, S., \& Breuer, C. (2014). Frühkindliche Sozialisation: Biologische, psychologische, linguistische, soziologische und ökonomische Perspektiven [Stellungnahme]. Halle: Deutsche Akademie der Naturforscher Leopoldina e. V. (Fehderführung), Nationale Akademie der Wissenschaften.

Wright, B.D., \& Stone, M.H. (1979). Best test design. Chicago: Mesa Press. http://www.sciencedirect. com/science/article/pii/B9780122381805500136

Yu, C.-Y. (2002). Evaluating cutoff criteria of model fit indices for latent variable models with binary and continuous outcomes. Bd. 30. Los Angeles: University of California. 